\title{
Low Complexity Partial Transmit Sequence with Complex Gain Memory Predistortion in OFDM Systems.
}

\begin{abstract}
In this paper the peak to average power ratio (PAPR) reduction and digital predistortion effects in orthogonal frequency division multiplexing (OFDM) systems are investigated. By applying a predistortion technique called complex gain memory predistortion (CGMP), power amplifier works at higher power efficiency. The proposed enhanced partial transmit sequence scheme is applied for PAPR reduction and integration with CGMP technique results in increasing in OFDM system efficiency and prolonged battery life. Simulation and results are examined with actual power amplifier and OFDM signal with quadrature phase shift keying (QPSK) modulation.
\end{abstract}

Keyword: Orthogonal frequency division multiplexing; Partial transmit sequence; PAPR; Power 